\title{
Sums of finite products of Bernoulli functions
}

Ravi P Agarwal', Dae San Kim², Taekyun Kim ${ }^{3,4^{*}}$ and Jongkyum Kwon ${ }^{5}$

"Correspondence: tkkim@kw.ac.kr ${ }^{3}$ Department of Mathematics, Kwangwoon University, Seoul, 139-701, Republic of Korea ${ }^{4}$ Department of Mathematics, College of Science, Tianjin Polytechnic University, Tianjin, 300160, China

Full list of author information is available at the end of the article

\begin{abstract}
In this paper, we consider three types of functions given by sums of finite products of Bernoulli functions and derive their Fourier series expansions. In addition, we express each of them in terms of Bernoulli functions.
\end{abstract}

MSC: $11 \mathrm{~B} 68 ; 42 \mathrm{~A} 16$

Keywords: Fourier series; sums of finite products of Bernoulli functions

\section{Introduction}

As is well known, the Bernoulli polynomials $B_{m}(x)$ are given by the generating function

$$
\frac{t}{e^{t}-1} e^{x t}=\sum_{m=0}^{\infty} B_{m}(x) \frac{t^{m}}{m !} \quad(\text { see }[1-10])
$$

When $x=0, B_{m}=B_{m}(0)$ are called Bernoulli numbers. For any real number $x$, we let

$$
\langle x\rangle=x-[x] \in[0,1)
$$

denote the fractional part of $x$.

Fourier series expansion of higher-order Bernoulli functions was treated in the recent paper [11]. Here we will consider the following three types of functions given by sums of finite products of Bernoulli functions and derive their Fourier series expansions. In addition, we will express each of them in terms of Bernoulli functions.

(1) $\alpha_{m}(\langle x\rangle)=\sum_{c_{1}+c_{2}+\cdots+c_{r}=m, c_{1}, \ldots, c_{r} \geq 0} B_{c_{1}}(\langle x\rangle) B_{c_{2}}(\langle x\rangle) \cdots B_{c_{r}}(\langle x\rangle)(m \geq 1)$;

(2) $\beta_{m}(\langle x\rangle)=\sum_{c_{1}+c_{2}+\cdots+c_{r}=m, c_{1}, \ldots, c_{r} \geq 0} \frac{1}{c_{1} ! c_{2} ! \cdots c_{r} !} B_{c_{1}}(\langle x\rangle) B_{c_{2}}(\langle x\rangle) \cdots B_{c_{r}}(\langle x\rangle)(m \geq 1)$;

(3) $\gamma_{r, m}(\langle x\rangle)=\sum_{c_{1}+c_{2}+\cdots+c_{r}=m, c_{1}, \ldots, c_{r} \geq 1} \frac{1}{c_{1} c_{2} \cdots c_{r}} B_{c_{1}}(\langle x\rangle) B_{c_{2}}(\langle x\rangle) \cdots B_{c_{r}}(\langle x\rangle)(m \geq r)$.

For elementary facts about Fourier analysis, the reader may refer to any book (for example, see $[12,13])$.

As to $\beta_{m}(\langle x\rangle)$, we note that the next polynomial identity follows immediately from Theorems 3.1 and 3.2, which is in turn derived from the Fourier series expansion of $\beta_{m}(\langle x\rangle)$ :

$$
\sum_{c_{1}+c_{2}+\cdots+c_{r}=m} \frac{1}{c_{1} ! c_{2} ! \cdots c_{r} !} B_{c_{1}}(x) B_{c_{2}}(x) \cdots B_{c_{r}}(x)=\frac{1}{r} \Omega_{m+1}+\sum_{j=1}^{m} \frac{r^{j-1}}{j !} \Omega_{m-j+1} B_{j}(x)
$$

(c) The Author(s) 2017. This article is distributed under the terms of the Creative Commons Attribution 4.0 International License (http://creativecommons.org/licenses/by/4.0/), which permits unrestricted use, distribution, and reproduction in any medium, provided you give appropriate credit to the original author(s) and the source, provide a link to the Creative Commons license, and indicate if changes were made. 
where

$$
\Omega_{l}=\sum_{\max \{0, r-l\} \leq a \leq r-1}\left(\begin{array}{l}
r \\
a
\end{array}\right) \sum_{c_{1}+c_{2}+\cdots+c_{a}=l+a-r} \frac{B_{c_{1}} B_{c_{2}} \cdots B_{c_{a}}}{c_{1} ! c_{2} ! \cdots c_{a} !} .
$$

The obvious polynomial identities can be derived also for $\alpha_{m}(\langle x\rangle)$ and $\gamma_{m}(\langle x\rangle)$ from Theorems 2.1 and 2.2, and Theorems 4.1 and 4.2, respectively. It is remarkable that from the Fourier series expansion of the function $\sum_{k=1}^{m-1} \frac{1}{k(m-k)} B_{k}(\langle x\rangle) B_{m-k}(\langle x\rangle)$ we can derive the Faber-Pandharipande-Zagier identity (see [14-16]) and the Miki identity (see [15-19]).

\section{The function $\alpha_{m}(\langle x\rangle)$}

Let $\alpha_{m}(x)=\sum_{c_{1}+c_{2}+\cdots+c_{r}=m} B_{c_{1}}(x) B_{c_{2}}(x) \cdots B_{c_{r}}(x)(m \geq 1)$. Here the sum runs over all nonnegative integers $c_{1}, c_{2}, \ldots, c_{r}$ with $c_{1}+c_{2}+\cdots+c_{r}=m(r \geq 1)$. Then we will consider the function

$$
\alpha_{m}(\langle x\rangle)=\sum_{c_{1}+c_{2}+\cdots+c_{r}=m} B_{c_{1}}(\langle x\rangle) B_{c_{2}}(\langle x\rangle) \cdots B_{c_{r}}(\langle x\rangle),
$$

defined on $(-\infty, \infty)$, which is periodic with period 1.

The Fourier series of $\alpha_{m}(\langle x\rangle)$ is

$$
\sum_{n=-\infty}^{\infty} A_{n}^{(m)} e^{2 \pi i n x}
$$

where

$$
\begin{aligned}
A_{n}^{(m)} & =\int_{0}^{1} \alpha_{m}(\langle x\rangle) e^{-2 \pi i n x} d x \\
& =\int_{0}^{1} \alpha_{m}(x) e^{-2 \pi i n x} d x .
\end{aligned}
$$

Before proceeding further, we need to observe the following.

$$
\begin{aligned}
\alpha_{m}^{\prime}(x)= & \sum_{c_{1}+c_{2}+\cdots+c_{r}=m}\left(c_{1} B_{c_{1}-1}(x) B_{c_{2}}(x) \cdots B_{c_{r}}(x)\right. \\
& \left.+\cdots+c_{r} B_{c_{1}}(x) B_{c_{2}}(x) \cdots B_{c_{r-1}}(x) B_{c_{r}-1}(x)\right) \\
= & \sum_{c_{1}+c_{2}+\cdots+c_{r}=m, c_{1} \geq 1} c_{1} B_{c_{1}-1}(x) B_{c_{2}}(x) \cdots B_{c_{r}}(x) \\
& +\cdots+\sum_{c_{1}+c_{2}+\cdots+c_{r}=m, c_{r} \geq 1} c_{r} B_{c_{1}-1}(x) B_{c_{2}}(x) \cdots B_{c_{r}}(x) \\
= & (m+r-1) \sum_{c_{1}+c_{2}+\cdots+c_{r}=m-1} B_{c_{1}}(x) B_{c_{2}}(x) \cdots B_{c_{r}}(x) \\
= & (m+r-1) \alpha_{m-1}(x) .
\end{aligned}
$$

From this, we have

$$
\left(\frac{\alpha_{m+1}(x)}{m+r}\right)^{\prime}=\alpha_{m}(x)
$$


and

$$
\int_{0}^{1} \alpha_{m}(x) d x=\frac{1}{m+r}\left(\alpha_{m+1}(1)-\alpha_{m+1}(0)\right) .
$$

For $m \geq 1$, we put

$$
\begin{aligned}
\Delta_{m} & =\alpha_{m}(1)-\alpha_{m}(0) \\
& =\sum_{c_{1}+c_{2}+\cdots+c_{r}=m}\left(B_{c_{1}}(1) B_{c_{2}}(1) \cdots B_{c_{r}}(1)-B_{c_{1}} B_{c_{2}} \cdots B_{c_{r}}\right) \\
& =\sum_{c_{1}+c_{2}+\cdots+c_{r}=m}\left(\left(B_{c_{1}}+\delta_{1, c_{1}}\right) \cdots\left(B_{c_{r}}+\delta_{1, c_{r}}\right)-B_{c_{1}} B_{c_{2}} \cdots B_{c_{r}}\right) \\
& =\sum_{\substack{0 \leq a \leq r \\
a \leq r-m}}\left(\begin{array}{l}
r \\
a
\end{array}\right)_{c_{1}+c_{2}+\cdots+c_{a}=m+a-r} \sum_{c_{1} B_{c_{2}} \cdots B_{c_{a}}-\sum_{c_{1}+c_{2}+\cdots+c_{r}=m} B_{c_{1}} B_{c_{2}} \cdots B_{c_{r}}}\left(\begin{array}{l}
r \\
a
\end{array}\right) \sum_{c_{1}+c_{2}+\cdots+c_{a}=m+a-r} B_{c_{1}} B_{c_{2}} \cdots B_{c_{a}},
\end{aligned}
$$

where we understand that, for $r-m \leq 0$ and $a=0$, the inner sum is $\delta_{m, r}$.

Observe here that the sum over all $c_{1}+c_{2}+\cdots+c_{r}=m$ of any term with $a$ of $B_{c_{e}}$ and $b$ of $\delta_{1, c_{f}}(1 \leq e, f \leq r, a+b=r)$, all give the same sum

$$
\begin{gathered}
\sum_{c_{1}+c_{2}+\cdots+c_{r}=m} B_{c_{1}} \cdots B_{c_{a}} \delta_{1, c_{a+1}} \cdots \delta_{1, c_{a+b}} \\
=\sum_{c_{1}+c_{2}+\cdots+c_{a}=m+a-r} B_{c_{1}} B_{c_{2}} \cdots B_{c_{a}}
\end{gathered}
$$

which is not an empty sum as long as $m+a-r \geq 0$, i.e., $a \geq r-m$.

Thus

$$
\alpha_{m}(1)=\alpha_{m}(0) \quad \Longleftrightarrow \quad \Delta_{m}=0
$$

and

$$
\int_{0}^{1} \alpha_{m}(x) d x=\frac{1}{m+r} \Delta_{m+1} .
$$

Now, we are ready to determine the Fourier coefficients $A_{n}^{(m)}$.

Case $1: n \neq 0$.

$$
\begin{aligned}
A_{n}^{(m)} & =\int_{0}^{1} \alpha_{m}(x) e^{-2 \pi i n x} d x \\
& =-\frac{1}{2 \pi i n}\left[\alpha_{m}(x) e^{-2 \pi i n x}\right]_{0}^{1}+\frac{1}{2 \pi i n} \int_{0}^{1} \alpha_{m}^{\prime}(x) e^{-2 \pi i n x} d x \\
& =\frac{m+r-1}{2 \pi i n} A_{n}^{(m-1)}-\frac{1}{2 \pi i n} \Delta_{m} \\
& =\frac{m+r-1}{2 \pi i n}\left(\frac{m+r-2}{2 \pi i n} A_{n}^{(m-2)}-\frac{1}{2 \pi i n} \Delta_{m-1}\right)-\frac{1}{2 \pi i n} \Delta_{m}
\end{aligned}
$$




$$
\begin{aligned}
& =\frac{(m+r-1)_{2}}{(2 \pi i n)^{2}} A_{n}^{(m-2)}-\sum_{j=0}^{2} \frac{(m+r-1)_{j-1}}{(2 \pi i n)^{j}} \Delta_{m-j+1} \\
& =\cdots \\
& =\frac{(m+r-1)_{m}}{(2 \pi i n)^{m}} A_{n}^{(0)}-\sum_{j=1}^{m} \frac{(m+r-1)_{j-1}}{(2 \pi i n)^{j}} \Delta_{m-j+1} \\
& =-\frac{1}{m+r} \sum_{j=1}^{m} \frac{(m+r)_{j}}{(2 \pi i n)^{j}} \Delta_{m-j+1},
\end{aligned}
$$

where $A_{n}^{(0)}=\int_{0}^{1} e^{-2 \pi i n x} d x=0$.

Case 2: $n=0$.

$$
A_{0}^{(m)}=\int_{0}^{1} \alpha_{m}(x) d x=\frac{1}{m+r} \Delta_{m+1} .
$$

Let us recall the following facts about Bernoulli functions $B_{m}(\langle x\rangle)$ :

(a) for $m \geq 2$,

$$
B_{m}(\langle x\rangle)=-m ! \sum_{\substack{n=-\infty \\ n \neq 0}}^{\infty} \frac{e^{2 \pi i n x}}{(2 \pi i n)^{m}}
$$

(b) for $m=1$,

$$
-\sum_{\substack{n=-\infty \\ n \neq 0}}^{\infty} \frac{e^{2 \pi i n x}}{2 \pi i n}= \begin{cases}B_{1}(\langle x\rangle) & \text { for } x \notin \mathbb{Z} \\ 0 & \text { for } x \in \mathbb{Z}\end{cases}
$$

$\alpha_{m}(\langle x\rangle)(m \geq 1)$ is piecewise $C^{\infty}$. Moreover, $\alpha_{m}(\langle x\rangle)$ is continuous for those positive integers $m$ with $\Delta_{m}=0$ and discontinuous with jump discontinuities at integers for those positive integers $m$ with $\Delta_{m} \neq 0$.

Assume first that $m$ is a positive integer with $\Delta_{m}=0$. Then $\alpha_{m}(1)=\alpha_{m}(0)$. Hence $\alpha_{m}(\langle x\rangle)$ is piecewise $C^{\infty}$ and continuous. Thus the Fourier series of $\alpha_{m}(\langle x\rangle)$ converges uniformly to $\alpha_{m}(\langle x\rangle)$, and

$$
\begin{aligned}
\alpha_{m}(\langle x\rangle)= & \frac{1}{m+r} \Delta_{m+1} \\
& +\sum_{n=-\infty, n \neq 0}^{\infty}\left(-\frac{1}{m+r} \sum_{j=1}^{m} \frac{(m+r)_{j}}{(2 \pi i n)^{j}} \Delta_{m-j+1}\right) e^{2 \pi i n x} \\
= & \frac{1}{m+r} \Delta_{m+1}+\frac{1}{m+r} \sum_{j=1}^{m}\left(\begin{array}{c}
m+r \\
j
\end{array}\right) \Delta_{m-j+1}\left(-j ! \sum_{\substack{n=-\infty \\
n \neq 0}}^{\infty} \frac{e^{2 \pi i n x}}{(2 \pi i n)^{j}}\right) \\
= & \frac{1}{m+r} \Delta_{m+1}+\frac{1}{m+r} \sum_{j=2}^{m}\left(\begin{array}{c}
m+r \\
j
\end{array}\right) \Delta_{m-j+1} B_{j}(\langle x\rangle) \\
& +\Delta_{m} \times\left\{\begin{array}{cc}
B_{1}(\langle x\rangle) & \text { for } x \notin \mathbb{Z}, \\
0 & \text { for } x \in \mathbb{Z} .
\end{array}\right.
\end{aligned}
$$


We can now state our first result.

Theorem 2.1 For each positive integer l, we let

$$
\Delta_{l}=\sum_{\max \{0, r-l) \leq a \leq r-1}\left(\begin{array}{l}
r \\
a
\end{array}\right) \sum_{c_{1}+c_{2}+\cdots+c_{a}=l+a-r} B_{c_{1}} B_{c_{2}} \cdots B_{c_{a}} .
$$

Assume that $\Delta_{m}=0$ for a positive integer $m$. Then we have the following.

(a) $\sum_{c_{1}+c_{2}+\cdots+c_{r}=m} B_{c_{1}}(\langle x\rangle) B_{c_{2}}(\langle x\rangle) \cdots B_{c_{r}}(\langle x\rangle)$ has the Fourier series expansion

$$
\begin{aligned}
& \sum_{c_{1}+c_{2}+\cdots+c_{r}=m} B_{c_{1}}(\langle x\rangle) B_{c_{2}}(\langle x\rangle) \cdots B_{c_{r}}(\langle x\rangle) \\
& =\frac{1}{m+r} \Delta_{m+1}-\sum_{\substack{n=-\infty \\
n \neq 0}}^{\infty}\left(\frac{1}{m+r} \sum_{j=1}^{m} \frac{(m+r)_{j}}{(2 \pi i n)^{j}} \Delta_{m-j+1}\right) e^{2 \pi i n x},
\end{aligned}
$$

for all $x \in \mathbb{R}$, where the convergence is uniform.

(b)

$$
\begin{aligned}
& \sum_{c_{1}+c_{2}+\cdots+c_{r}=m} B_{c_{1}}(\langle x\rangle) B_{c_{2}}(\langle x\rangle) \cdots B_{c_{r}}(\langle x\rangle) \\
& =\frac{1}{m+r} \Delta_{m+1}+\frac{1}{m+r} \sum_{j=2}^{m}\left(\begin{array}{c}
m+r \\
j
\end{array}\right) \Delta_{m-j+1} B_{j}(\langle x\rangle),
\end{aligned}
$$

for all $x \in \mathbb{R}$, where $B_{j}(\langle x\rangle)$ is the Bernoulli function.

Assume next that $\Delta_{m} \neq 0$ for a positive integer $m$. Then $\alpha_{m}(1) \neq \alpha_{m}(0)$. Hence $\alpha_{m}(\langle x\rangle)$ is piecewise $C^{\infty}$ and discontinuous with jump discontinuities at integers. The Fourier series of $\alpha_{m}(\langle x\rangle)$ converges pointwise to $\alpha_{m}(\langle x\rangle)$ for $x \notin \mathbb{Z}$ and converges to

$$
\frac{1}{2}\left(\alpha_{m}(0)+\alpha_{m}(1)\right)=\alpha_{m}(0)+\frac{1}{2} \Delta_{m}
$$

for $x \in \mathbb{Z}$.

Now, we can state our second result.

Theorem 2.2 For each positive integer l, we let

$$
\Delta_{l}=\sum_{\max \{0, r-l\} \leq a \leq r-1}\left(\begin{array}{l}
r \\
a
\end{array}\right) \sum_{c_{1}+c_{2}+\cdots+c_{a}=l+a-r} B_{c_{1}} B_{c_{2}} \cdots B_{c_{a}} .
$$

Assume that $\Delta_{m} \neq 0$ for a positive integer $m$. Then we have the following.

$$
\text { (a) } \begin{aligned}
\frac{1}{m+r} \Delta_{m+1}+\sum_{\substack{n=-\infty \\
n \neq 0}}^{\infty}\left(-\frac{1}{m+r} \sum_{j=1}^{m} \frac{(m+r)_{j}}{(2 \pi i n)^{j}} \Delta_{m-j+1}\right) e^{2 \pi i n x} \\
\quad= \begin{cases}\sum_{c_{1}+c_{2}+\cdots+c_{r}=m} B_{c_{1}}(\langle x\rangle) B_{c_{2}}(\langle x\rangle) \cdots B_{c_{r}}(\langle x\rangle) & \text { for } x \notin \mathbb{Z}, \\
\sum_{c_{1}+c_{2}+\cdots+c_{r}=m} B_{c_{1}} B_{c_{2}} \cdots B_{c_{r}}+\frac{1}{2} \Delta_{m} & \text { for } x \in \mathbb{Z} .\end{cases}
\end{aligned}
$$


(b) $\frac{1}{m+r} \Delta_{m+1}+\frac{1}{m+r} \sum_{j=1}^{m}\left(\begin{array}{c}m+r \\ j\end{array}\right) \Delta_{m-j+1} B_{j}(\langle x\rangle)$

$$
\begin{gathered}
=\sum_{c_{1}+c_{2}+\cdots+c_{r}=m} B_{c_{1}}(\langle x\rangle) B_{c_{2}}(\langle x\rangle) \cdots B_{c_{r}}(\langle x\rangle) \quad \text { for } x \notin \mathbb{Z} ; \\
\frac{1}{m+r} \Delta_{m+1}+\frac{1}{m+r} \sum_{j=2}^{m}\left(\begin{array}{c}
m+r \\
j
\end{array}\right) \Delta_{m-j+1} B_{j}(\langle x\rangle) \\
=\sum_{c_{1}+c_{2}+\cdots+c_{r}=m} B_{c_{1}} B_{c_{2}} \cdots B_{c_{r}}+\frac{1}{2} \Delta_{m} \quad \text { for } x \in \mathbb{Z} .
\end{gathered}
$$

\section{The function $\beta_{m}(\langle x\rangle)$}

Let $\beta_{m}(x)=\sum_{c_{1}+c_{2}+\cdots+c_{r}=m} \frac{1}{c_{1} ! c_{2} ! \cdots c_{r} !} B_{c_{1}}(x) B_{c_{2}}(x) \cdots B_{c_{r}}(x)(m \geq 1)$. Here the sum runs over all nonnegative integers $c_{1}, c_{2}, \ldots, c_{r}$ with $c_{1}+c_{2}+\cdots+c_{r}=m(r \geq 1)$. Then we will consider the function

$$
\beta_{m}(\langle x\rangle)=\sum_{c_{1}+c_{2}+\cdots+c_{r}=m} \frac{1}{c_{1} ! c_{2} ! \cdots c_{r} !} B_{c_{1}}(\langle x\rangle) B_{c_{2}}(\langle x\rangle) \cdots B_{c_{r}}(\langle x\rangle),
$$

defined on $(-\infty, \infty)$, which is periodic with period 1 . The Fourier series of $\beta_{m}(\langle x\rangle)$ is

$$
\sum_{n=-\infty}^{\infty} B_{n}^{(m)} e^{2 \pi i n x}
$$

where

$$
B_{n}^{(m)}=\int_{0}^{1} \beta_{m}(\langle x\rangle) e^{-2 \pi i n x} d x=\int_{0}^{1} \beta_{m}(x) e^{-2 \pi i n x} d x .
$$

Before proceeding further, we need to observe the following.

$$
\begin{aligned}
\beta_{m}^{\prime}(x)= & \sum_{c_{1}+c_{2}+\cdots+c_{r}=m}\left(\frac{c_{1}}{c_{1} ! c_{2} ! \cdots c_{r} !} B_{c_{1}-1}(x) B_{c_{2}}(x) \cdots B_{c_{r}}(x)\right. \\
& \left.+\cdots+\frac{c_{r}}{c_{1} ! c_{2} ! \cdots c_{r} !} B_{c_{1}}(x) B_{c_{2}}(x) \cdots B_{c_{r}-1}(x)\right) \\
= & \sum_{c_{1}+c_{2}+\cdots+c_{r}=m, c_{1} \geq 1} \frac{1}{\left(c_{1}-1\right) ! c_{2} ! \cdots c_{r} !} B_{c_{1}-1}(x) B_{c_{2}}(x) \cdots B_{c_{r}}(x) \\
& +\cdots+\sum_{c_{1}+c_{2}+\cdots+c_{r}=m, c_{r} \geq 1} \frac{1}{c_{1} ! c_{2} ! \cdots\left(c_{r}-1\right) !} B_{c_{1}}(x) B_{c_{2}}(x) \cdots B_{c_{r}-1}(x) \\
= & r \sum_{c_{1}+c_{2}+\cdots+c_{r}=m-1} \frac{1}{c_{1} ! c_{2} ! \cdots c_{r} !} B_{c_{1}}(x) B_{c_{2}}(x) \cdots B_{c_{r}}(x) \\
= & r \beta_{m-1}(x) .
\end{aligned}
$$

From this, we have

$$
\left(\frac{\beta_{m+1}(x)}{r}\right)^{\prime}=\beta_{m}(x)
$$


and

$$
\int_{0}^{1} \beta_{m}(x) d x=\frac{1}{r}\left(\beta_{m+1}(1)-\beta_{m+1}(0)\right) .
$$

Let

$$
\begin{aligned}
\Omega_{m}= & \beta_{m}(1)-\beta_{m}(0) \\
= & \sum_{c_{1}+c_{2}+\cdots+c_{r}=m} \frac{B_{c_{1}}(1) B_{c_{2}}(1) \cdots B_{c_{r}}(1)}{c_{1} ! c_{2} ! \cdots c_{r} !}-\sum_{c_{1}+c_{2}+\cdots+c_{r}=m} \frac{B_{c_{1}} B_{c_{2}} \cdots B_{c_{r}}}{c_{1} ! c_{2} ! \cdots c_{r} !} \\
= & \sum_{c_{1}+c_{2}+\cdots+c_{r}=m} \frac{\left(B_{c_{1}}+\delta_{1, c_{1}}\right)\left(B_{c_{2}}+\delta_{1, c_{2}}\right) \cdots\left(B_{c_{r}}+\delta_{1, c_{r}}\right)}{c_{1} ! c_{2} ! \cdots c_{r} !} \\
& -\sum_{c_{1}+c_{2}+\cdots+c_{r}=m} \frac{B_{c_{1}} B_{c_{2}} \cdots B_{c_{r}}}{c_{1} ! c_{2} ! \cdots c_{r} !} \\
= & \sum_{\max \{0, r-m\} \leq a \leq r-1}\left(\begin{array}{l}
r \\
a
\end{array}\right)_{c_{1}+c_{2}+\cdots+c_{a}=m+a-r} \frac{B_{c_{1}} B_{c_{2}} \cdots B_{c_{a}}}{c_{1} ! c_{2} ! \cdots c_{a} !},
\end{aligned}
$$

where we understand that, for $r-m \leq 0$ and $a=0$, the inner sum is $\delta_{m, r}$.

Observe here that the sum over all $c_{1}+c_{2}+\cdots+c_{r}=m$ of any term with $a$ of $B_{c_{e}}$ and $b$ of $\delta_{1, c_{f}}(1 \leq e, f \leq r, a+b=r)$, all give the same sum

$$
\begin{gathered}
\sum_{c_{1}+c_{2}+\cdots+c_{r}=m} \frac{B_{c_{1}} \cdots B_{c_{a}} \delta_{1, c_{a+1}} \cdots \delta_{1, c_{a+b}}}{c_{1} ! c_{2} ! \cdots c_{r} !} \\
=\sum_{c_{1}+c_{2}+\cdots+c_{a}=m+a-r} \frac{B_{c_{1}} B_{c_{2}} \cdots B_{c_{r}}}{c_{1} ! c_{2} ! \cdots c_{r} !}
\end{gathered}
$$

which is not an empty sum as long as $m+a-r \geq 0$, i.e., $a \geq r-m$.

Also, we have

$$
\beta_{m}(1)=\beta_{m}(0) \quad \Leftrightarrow \quad \Omega_{m}=0
$$

and

$$
\int_{0}^{1} \beta_{m}(x) d x=\frac{1}{r} \Omega_{m+1} .
$$

Now, we would like to determine the Fourier coefficients $B_{n}^{(m)}$.

Case $1: n \neq 0$.

$$
\begin{aligned}
B_{n}^{(m)} & =\int_{0}^{1} \beta_{m}(x) e^{-2 \pi i n x} d x \\
& =-\frac{1}{2 \pi i n}\left[\beta_{m}(x) e^{-2 \pi i n x}\right]_{0}^{1}+\frac{1}{2 \pi i n} \int_{0}^{1} \beta_{m}^{\prime}(x) e^{-2 \pi i n x} d x \\
& =-\frac{1}{2 \pi i n}\left(\beta_{m}(1)-\beta_{m}(0)\right)+\frac{r}{2 \pi i n} \int_{0}^{1} \beta_{m-1}(x) e^{-2 \pi i n x} d x
\end{aligned}
$$




$$
\begin{aligned}
& =\frac{r}{2 \pi i n} B_{n}^{(m-1)}-\frac{1}{2 \pi i n} \Omega_{m} \\
& =\frac{r}{2 \pi i n}\left(\frac{r}{2 \pi i n} B_{n}^{(m-2)}-\frac{1}{2 \pi i n} \Omega_{m-1}\right)-\frac{1}{2 \pi i n} \Omega_{m} \\
& =\left(\frac{r}{2 \pi i n}\right)^{2} B_{n}^{(m-2)}-\sum_{j=1}^{2} \frac{r^{j-1}}{(2 \pi i n)^{j}} \Omega_{m-j+1} \\
& =\cdots \\
& =\left(\frac{r}{2 \pi i n}\right)^{m} B_{n}^{(0)}-\sum_{j=1}^{m} \frac{r^{j-1}}{(2 \pi i n)^{j}} \Omega_{m-j+1} \\
& =-\sum_{j=1}^{m} \frac{r^{j-1}}{(2 \pi i n)^{j}} \Omega_{m-j+1}
\end{aligned}
$$

where $B_{n}^{(0)}=\int_{0}^{1} e^{-2 \pi i n x} d x=0$.

Case 2: $n=0$.

$$
B_{0}^{(m)}=\int_{0}^{1} \beta_{m}(x)=\frac{1}{r} \Omega_{m+1}
$$

$\beta_{m}(\langle x\rangle)(m \geq 1)$ is piecewise $C^{\infty}$. Moreover, $\beta_{m}(\langle x\rangle)$ is continuous for those positive integers $m$ with $\Omega_{m}=0$ and discontinuous with jump discontinuities at integers for those positive integers $m$ with $\Omega_{m} \neq 0$.

Assume first that $\Omega_{m}=0$ for a positive integer $m$. Then $\beta_{m}(1)=\beta_{m}(0)$. Hence $\beta_{m}(\langle x\rangle)$ is piecewise $C^{\infty}$ and continuous. Thus the Fourier series of $\beta_{m}(\langle x\rangle)$ converges uniformly to $\beta_{m}(\langle x\rangle)$, and

$$
\begin{aligned}
\beta_{m}(\langle x\rangle)= & \frac{1}{r} \Omega_{m+1}+\sum_{\substack{n=-\infty \\
n \neq 0}}^{\infty}\left(-\sum_{j=1}^{m} \frac{r^{j-1}}{(2 \pi i n)^{j}} \Omega_{m-j+1}\right) e^{2 \pi i n x} \\
= & \frac{1}{r} \Omega_{m+1}+\sum_{j=1}^{m} \frac{r^{j-1}}{j !} \Omega_{m-j+1} \times\left(-j ! \sum_{\substack{n=-\infty \\
n \neq 0}}^{\infty} \frac{e^{2 \pi i n x}}{(2 \pi i n)^{j}}\right) \\
= & \frac{1}{r} \Omega_{m+1}+\sum_{\substack{j=2 \\
m}}^{m} \frac{r^{j-1}}{j !} \Omega_{m-j+1} B_{j}(\langle x\rangle) \\
& +\Omega_{m} \times \begin{cases}B_{1}(\langle x\rangle) & \text { for } x \notin \mathbb{Z}, \\
0 & \text { for } x \in \mathbb{Z} .\end{cases}
\end{aligned}
$$

Now, we can state our first result.

Theorem 3.1 For each positive integer l, we let

$$
\Omega_{l}=\sum_{\max \{0, r-l\} \leq a \leq r-1}\left(\begin{array}{l}
r \\
a
\end{array}\right) \sum_{c_{1}+c_{2}+\cdots+c_{a}=l+a-r} \frac{B_{c_{1}} B_{c_{2}} \cdots B_{c_{a}}}{c_{1} ! c_{2} ! \cdots c_{a} !} .
$$

Assume that $\Omega_{m}=0$ for a positive integer $m$. Then we have the following. 
(a) $\sum_{c_{1}+c_{2}+\cdots+c_{r}=m} \frac{1}{c_{1} ! c_{2} ! \cdots c_{r} !} B_{c_{1}}(\langle x\rangle) B_{c_{2}}(\langle x\rangle) \cdots B_{c_{r}}(\langle x\rangle)$ has the Fourier series expansion

$$
\begin{gathered}
\sum_{c_{1}+c_{2}+\cdots+c_{r}=m} \frac{1}{c_{1} ! c_{2} ! \cdots c_{r} !} B_{c_{1}}(\langle x\rangle) B_{c_{2}}(\langle x\rangle) \cdots B_{c_{r}}(\langle x\rangle) \\
=\frac{1}{r} \Omega_{m+1}-\sum_{\substack{n=-\infty \\
n \neq 0}}^{\infty}\left(\sum_{j=1}^{m} \frac{r^{j-1}}{(2 \pi i n)^{j}} \Omega_{m-j+1}\right) e^{2 \pi i n x},
\end{gathered}
$$

(b)

for all $x \in(-\infty, \infty)$, where the convergence is uniform.

$$
\begin{aligned}
& \sum_{c_{1}+c_{2}+\cdots+c_{r}=m} \frac{1}{c_{1} ! c_{2} ! \cdots c_{r} !} B_{c_{1}}(\langle x\rangle) B_{c_{2}}(\langle x\rangle) \cdots B_{c_{r}}(\langle x\rangle) \\
& =\frac{1}{r} \Omega_{m+1}+\sum_{j=2}^{m} \frac{r^{j-1}}{j !} \Omega_{m-j+1} B_{j}(\langle x\rangle),
\end{aligned}
$$

for all $x \in(-\infty, \infty)$, where $B_{j}(\langle x\rangle)$ is the Bernoulli function.

Assume next that $m$ is a positive integer with $\Omega_{m} \neq 0$. Then $\beta_{m}(1) \neq \beta_{m}(0)$. Hence $\beta_{m}(\langle x\rangle)$ is piecewise $C^{\infty}$ and discontinuous with jump discontinuities at integers. Thus the Fourier series of $\beta_{m}(\langle x\rangle)$ converges pointwise to $\beta_{m}(\langle x\rangle)$ for $x \notin \mathbb{Z}$ and converges to

$$
\begin{aligned}
\frac{1}{2}\left(\beta_{m}(0)+\beta_{m}(1)\right) & =\beta_{m}(0)+\frac{1}{2} \Omega_{m} \\
& =\sum_{c_{1}+c_{2}+\cdots+c_{r}=m} \frac{1}{c_{1} ! c_{2} ! \cdots c_{r} !} B_{c_{1}} B_{c_{2}} \cdots B_{c_{r}}+\frac{1}{2} \Omega_{m}
\end{aligned}
$$

for $x \in \mathbb{Z}$.

Now, we can state our second result.

Theorem 3.2 For each positive integer l, let

$$
\Omega_{l}=\sum_{\max \{0, r-l\} \leq a \leq r-1}\left(\begin{array}{l}
r \\
a
\end{array}\right) \sum_{c_{1}+c_{2}+\cdots+c_{a}=l+a-r} \frac{B_{c_{1}} B_{c_{2}} \cdots B_{c_{a}}}{c_{1} ! c_{2} ! \cdots c_{a} !} .
$$

Assume that $\Omega_{m} \neq 0$ for a positive integer $m$. Then we have the following.

$$
\text { (a) } \begin{aligned}
\frac{1}{r} \Omega_{m+1}-\sum_{n=-\infty, n \neq 0}^{\infty}\left(\sum_{j=1}^{m} \frac{r^{j-1}}{(2 \pi i n)^{j}} \Omega_{m-j+1}\right) e^{2 \pi i n x} \\
\quad= \begin{cases}\sum_{c_{1}+c_{2}+\cdots+c_{r}=m} \frac{1}{c_{1} ! c_{2} ! \cdots c_{r} !} B_{c_{1}}(\langle x\rangle) B_{c_{2}}(\langle x\rangle) \cdots B_{c_{r}}(\langle x\rangle) & \text { for } x \notin \mathbb{Z}, \\
\sum_{c_{1}+c_{2}+\cdots+c_{r}=m} \frac{1}{c_{1} ! c_{2} ! \cdots c_{r} !} B_{c_{1}} B_{c_{2}} \cdots B_{c_{r}}+\frac{1}{2} \Omega_{m} & \text { for } x \in \mathbb{Z} .\end{cases}
\end{aligned}
$$

Here the convergence is pointwise.

$$
\begin{aligned}
& \text { (b) } \frac{1}{r} \Omega_{m+1}+\sum_{j=1}^{m} \frac{r^{j-1}}{j !} \Omega_{m-j+1} B_{j}(\langle x\rangle) \\
& =\sum_{c_{1}+c_{2}+\cdots+c_{r}=m} \frac{1}{c_{1} ! c_{2} ! \cdots c_{r} !} B_{c_{1}}(\langle x\rangle) B_{c_{2}}(\langle x\rangle) \cdots B_{c_{r}}(\langle x\rangle) \text { for } x \notin \mathbb{Z},
\end{aligned}
$$




$$
\begin{aligned}
& \frac{1}{r} \Omega_{m+1}+\sum_{j=2}^{m} \frac{r^{j-1}}{j !} \Omega_{m-j+1} B_{j}(\langle x\rangle) \\
& \quad=\sum_{c_{1}+c_{2}+\cdots+c_{r}=m} \frac{1}{c_{1} ! c_{2} ! \cdots c_{r} !} B_{c_{1}} B_{c_{2}} \cdots B_{c_{r}}+\frac{1}{2} \Omega_{m} \quad \text { for } x \in \mathbb{Z} .
\end{aligned}
$$

Here $B_{j}(\langle x\rangle)$ is the Bernoulli function.

\section{The function $\gamma_{r, m}(\langle x\rangle)$}

Let $\gamma_{r, m}(x)=\sum_{c_{1}+c_{2}+\cdots+c_{r}=m, c_{1}, \ldots, c_{r} \geq 1} \frac{1}{c_{1} c_{2} \cdots c_{r}} B_{c_{1}}(x) B_{c_{2}}(x) \cdots B_{c_{r}}(x)(m \geq r \geq 1)$. Here the sum is over all positive integers $c_{1}, c_{2}, \ldots, c_{r}$ with $c_{1}+c_{2}+\cdots+c_{r}=m$.

$$
\begin{aligned}
\gamma_{r, m}^{\prime}(x)= & \sum_{c_{1}+c_{2}+\cdots+c_{r}=m, c_{1}, \ldots, c_{r} \geq 1} \frac{1}{c_{2} \cdots c_{r}} B_{c_{1}-1}(x) B_{c_{2}}(x) \cdots B_{c_{r}}(x) \\
& +\sum_{c_{1}+c_{2}+\cdots+c_{r}=m, c_{1}, \ldots, c_{r} \geq 1} \frac{1}{c_{1} c_{3} \cdots c_{r}} B_{c_{1}}(x) B_{c_{2}-1}(x) \cdots B_{c_{r}}(x) \\
& +\cdots+\sum_{c_{1}+c_{2}+\cdots+c_{r}=m, c_{1}, \ldots, c_{r} \geq 1} \frac{1}{c_{1} c_{2} \cdots c_{r-1}} B_{c_{1}}(x) B_{c_{2}}(x) \cdots B_{c_{r}-1}(x) \\
= & \sum_{c_{2}+\cdots+c_{r}=m-1, c_{2}, \ldots, c_{r} \geq 1} \frac{1}{c_{2} \cdots c_{r}} B_{c_{2}}(x) \cdots B_{c_{r}}(x) \\
& +\sum_{c_{1}+\cdots+c_{r}=m-1, c_{1}, \ldots, c_{r} \geq 1} \frac{1}{c_{2} \cdots c_{r}} B_{c_{1}}(x) \cdots B_{c_{r}}(x) \\
& +\cdots+\sum_{c_{1}+c_{2}+\cdots+c_{r-1}=m-1, c_{1}, \ldots, c_{r-1} \geq 1} \frac{1}{c_{1} c_{2} \cdots c_{r-1}} B_{c_{1}}(x) \cdots B_{c_{r-1}}(x) \\
& +\sum_{c_{1}+c_{2}+\cdots+c_{r}=m-1, c_{1}, \ldots, c_{r} \geq 1} \frac{1}{c_{1} c_{2} \cdots c_{r-1}} B_{c_{1}}(x) \cdots B_{c_{r}}(x) \\
= & r \gamma_{r-1, m-1}(x)+(m-1) \gamma_{r, m-1}(x) .
\end{aligned}
$$

Thus,

$$
\gamma_{r, m}^{\prime}(x)=r \gamma_{r-1, m-1}(x)+(m-1) \gamma_{r, m-1}(x) \quad(m \geq r)
$$

with $\gamma_{r, r-1}(x)=0$.

Replacing $m$ by $m+1$, we get

$$
m \gamma_{r, m}(x)=\gamma_{r, m+1}^{\prime}(x)-r \gamma_{r-1, m}(x) .
$$

Denoting $\int_{0}^{1} \gamma_{r, m}(x) d x$ by $a_{r, m}$, we have

$$
a_{r, m}=-\frac{r}{m} a_{r-1, m}+\frac{1}{m} \Lambda_{r, m+1}
$$

where $\Lambda_{r, m}=\gamma_{r, m}(1)-\gamma_{r, m}(0)$. From the recurrence relation (4.4), we can easily show that

$$
\int_{0}^{1} \gamma_{r, m}(x) d x=\sum_{j=1}^{r-1}(-1)^{j-1} \frac{(r)_{j-1}}{m^{j}} \Lambda_{r-j+1, m+1}
$$




$$
\begin{aligned}
\Lambda_{r, m}= & \gamma_{r, m}(1)-\gamma_{r, m}(0) \\
= & \sum_{c_{1}+c_{2}+\cdots+c_{r}=m, c_{1}, \ldots, c_{r} \geq 1} \frac{B_{c_{1}}(1) \cdots B_{c_{r}}(1)}{c_{1} \cdots c_{r}} \\
& -\sum_{c_{1}+c_{2}+\cdots+c_{r}=m, c_{1}, \ldots, c_{r} \geq 1} \frac{B_{c_{1}} \cdots B_{c_{r}}}{c_{1} \cdots c_{r}} \\
= & \sum_{c_{1}+c_{2}+\cdots+c_{r}=m, c_{1}, \ldots, c_{r} \geq 1}\left(B_{c_{1}}+\delta_{1, c_{1}}\right) \cdots\left(B_{c_{r}}+\delta_{1, c_{r}}\right) \\
& -\sum_{c_{1}+c_{2}+\cdots+c_{r}=m, c_{1}, \ldots, c_{r} \geq 1} \frac{B_{c_{1}} \cdots B_{c_{r}}}{c_{1} \cdots c_{r}} \\
= & \sum_{0 \leq a \leq r-1}\left(\begin{array}{l}
r \\
a
\end{array}\right)_{c_{1}+c_{2}+\cdots+c_{a}=m+a-r, c_{1}, \ldots, c_{a} \geq 1} \frac{B_{c_{1}} B_{c_{2}} \cdots B_{c_{a}}}{c_{1} c_{2} \cdots c_{a}} .
\end{aligned}
$$

Observe here that the sum over all positive integers $c_{1}, \ldots, c_{r}$ satisfying $c_{1}+c_{2}+\cdots+c_{r}=$ $m$ of any term with $a$ of $B_{c_{e}}$ and $b$ of $\delta_{1, c_{f}}(1 \leq e, f \leq r, a+b=r)$, all give the same sum

$$
\begin{gathered}
\sum_{c_{1}+c_{2}+\cdots+c_{r}=m, c_{1}, \ldots, c_{a} \geq 1} \frac{B_{c_{1}} \cdots B_{c_{a}} \delta_{1, c_{a+1}} \cdots \delta_{1, c_{a+b}}}{c_{1} c_{2} \cdots c_{r}} \\
=\sum_{c_{1}+c_{2}+\cdots+c_{a}=m+a-r, c_{1}, \ldots, c_{a} \geq 1} \frac{B_{c_{1}} B_{c_{2}} \cdots B_{c_{a}}}{c_{1} c_{2} \cdots c_{a}}
\end{gathered}
$$

and that, as $m+a-r \geq a$, there are no empty sums.

Here we note that, for $a=0$, the inner sum is $\delta_{m, r}$ since it corresponds to the sums

$$
\sum_{c_{1}+c_{2}+\cdots+c_{r}=m, c_{1}, \ldots, c_{r} \geq 1} \frac{\delta_{1, c_{1}} \delta_{1, c_{2}} \cdots \delta_{1, c_{r}}}{c_{1} c_{2} \cdots c_{r}} .
$$

Also, $\gamma_{r, m}(1)=\gamma_{r, m}(0) \Leftrightarrow \Lambda_{r, m}=0$.

Now, we would like to consider the function

$$
\gamma_{r, m}(\langle x\rangle)=\sum_{c_{1}+c_{2}+\cdots+c_{r}=m, c_{1}, \ldots, c_{r} \geq 1} \frac{1}{c_{1} c_{2} \cdots c_{r}} B_{c_{1}}(\langle x\rangle) B_{c_{2}}(\langle x\rangle) \cdots B_{c_{r}}(\langle x\rangle),
$$

defined on $(-\infty, \infty)$, which is periodic with period 1 .

The Fourier series of $\gamma_{r, m}(\langle x\rangle)$ is

$$
\sum_{n=-\infty}^{\infty} C_{n}^{(r, m)} e^{2 \pi i n x}
$$

where

$$
C_{n}^{(r, m)}=\int_{0}^{1} \gamma_{r, m}(\langle x\rangle) e^{-2 \pi i n x} d x=\int_{0}^{1} \gamma_{r, m}(x) e^{-2 \pi i n x} d x .
$$

Now, we are going to determine the Fourier coefficients $C_{n}^{(r, m)}$. 
Case $1: n \neq 0$.

$$
\begin{aligned}
C_{n}^{(r, m)}= & \int_{0}^{1} \gamma_{r, m}(x) e^{-2 \pi i n x} d x \\
= & -\frac{1}{2 \pi i n}\left[\gamma_{r, m}(x) e^{-2 \pi i n x}\right]_{0}^{1}+\frac{1}{2 \pi i n} \int_{0}^{1} \gamma_{r, m}^{\prime}(x) e^{-2 \pi i n x} d x \\
= & -\frac{1}{2 \pi i n}\left(\gamma_{r, m}(1)-\gamma_{r, m}(0)\right)+\frac{1}{2 \pi i n} \int_{0}^{1}\left(r \gamma_{r-1, m-1}(x)+(m-1) \gamma_{r, m-1}(x)\right) \\
& \times e^{-2 \pi i n x} d x \\
= & -\frac{1}{2 \pi i n} \Lambda_{r, m}+\frac{1}{2 \pi i n} C_{n}^{(r, m-1)}+\frac{r}{2 \pi i n} C_{n}^{(r-1, m-1)} .
\end{aligned}
$$

From this, we obtain

$$
\begin{aligned}
C_{n}^{(r, m)}= & \frac{m-1}{2 \pi i n} C_{n}^{(r, m-1)}+\frac{r}{2 \pi i n} C_{n}^{(r-1, m-1)}-\frac{1}{2 \pi i n} \Lambda_{r, m} \\
= & \frac{m-1}{2 \pi i n}\left(\frac{m-2}{2 \pi i n} C_{n}^{(r, m-2)}+\frac{r}{2 \pi i n} C_{n}^{(r-1, m-2)}-\frac{1}{2 \pi i n} \Lambda_{r, m-1}\right) \\
& +\frac{r}{2 \pi i n} C_{n}^{(r-1, m-1)}-\frac{1}{2 \pi i n} \Lambda_{r, m} \\
= & \frac{(m-1)_{2}}{(2 \pi i n)^{2}} C_{n}^{(r, m-2)}+\sum_{j=1}^{2} \frac{r(m-1)_{j-1}}{(2 \pi i n)^{j}} C_{n}^{(r-1, m-j)}-\sum_{j=1}^{2} \frac{(m-1)_{j-1}}{(2 \pi i n)^{j}} \Lambda_{r, m-j+1} \\
= & \cdots \\
= & \frac{(m-1)_{m-r}}{(2 \pi i n)^{m-r}} C_{n}^{(r, r)}+\sum_{j=1}^{m-r} \frac{r(m-1)_{j-1}}{(2 \pi i n)^{j}} C_{n}^{(r-1, m-j)}-\sum_{j=1}^{m-r} \frac{(m-1)_{j-1}}{(2 \pi i n)^{j}} \Lambda_{r, m-j+1} .
\end{aligned}
$$

Here,

$$
\begin{aligned}
C_{n}^{(r, r)} & =\int_{0}^{1}\left(x-\frac{1}{2}\right)^{r} e^{-2 \pi i n x} d x \\
& =-\frac{1}{2 \pi i n}\left[\left(x-\frac{1}{2}\right)^{r} e^{-2 \pi i n x}\right]_{0}^{1}+\frac{r}{2 \pi i n} \int_{0}^{1}\left(x-\frac{1}{2}\right)^{r-1} e^{-2 \pi i n x} d x \\
& =-\frac{1}{2 \pi i n}\left(\left(\frac{1}{2}\right)^{r}-\left(-\frac{1}{2}\right)^{r}\right)+\frac{r}{2 \pi i n} C_{n}^{(r-1, r-1)},
\end{aligned}
$$

and

$$
\Lambda_{r, r}=\gamma_{r, r}(1)-\gamma_{r, r}(0)=\left(\frac{1}{2}\right)^{r}-\left(-\frac{1}{2}\right)^{r} .
$$

Thus

$$
C_{n}^{(r, r)}=-\frac{1}{2 \pi i n} \Lambda_{r, r}+\frac{r}{2 \pi i n} C_{n}^{(r-1, r-1)} .
$$

Finally, we obtain, for $n \neq 0$,

$$
C_{n}^{(r, m)}=\sum_{j=1}^{m-r+1} \frac{r(m-1)_{j-1}}{(2 \pi i n)^{j}} C_{n}^{(r-1, m-j)}-\sum_{j=1}^{m-r+1} \frac{(m-1)_{j-1}}{(2 \pi i n)^{j}} \Lambda_{r, m-j+1} .
$$


Also, we note that, for $n \neq 0$,

$$
C_{n}^{(1, m)}=\frac{1}{m} \int_{0}^{1} B_{m}(x) e^{-2 \pi i n x} d x=-\frac{(m-1) !}{(2 \pi i n)^{m}}
$$

Thus, for $n \neq 0$, (4.17) together with (4.18) determine all $C_{n}^{(r, m)}$ recursively.

Case 2: $n=0$.

$$
C_{0}^{(r, m)}=\int_{0}^{1} \gamma_{r, m}(x) d x=\sum_{j=1}^{r}(-1)^{j-1} \frac{(r)_{j-1}}{m^{j}} \Lambda_{r-j+1, m+1} .
$$

$\gamma_{r, m}(\langle x\rangle)(m \geq r \geq 1)$ is piecewise $C^{\infty}$. In addition, $\gamma_{r, m}(\langle x\rangle)$ is continuous for those positive integers $r, m$ with $\Lambda_{r, m}=0$ and discontinuous with jump discontinuities at integers for those positive integers $r, m$ with $\Lambda_{r, m} \neq 0$.

Assume first that $\Lambda_{r, m}=0$ for some integers $r, m$ with $m \geq r \geq 1$. Then $\gamma_{r, m}(1)=\gamma_{r, m}(0)$. Hence $\gamma_{r, m}(\langle x\rangle)$ is piecewise $C^{\infty}$ and continuous. Thus the Fourier series of $\gamma_{m}(\langle x\rangle)$ converges uniformly to $\gamma_{m}(\langle x\rangle)$, and

$$
\gamma_{m}(\langle x\rangle)=C_{0}^{(r, m)}+\sum_{\substack{n=-\infty \\ n \neq 0}}^{\infty} C_{n}^{(r, m)} e^{2 \pi i n x}
$$

where $C_{0}^{(r, m)}$ is given by (4.19), and $C_{n}^{(r, m)}$, for each $n \neq 0$, are determined by relations (4.17) and (4.18).

Now, we are ready to state our first theorem.

Theorem 4.1 For all integers $s, l$, with $l \geq s \geq 1$, we let

$$
\begin{aligned}
\Lambda_{s, l} & =\sum_{0 \leq a \leq s-1}\left(\begin{array}{l}
s \\
a
\end{array}\right) \sum_{c_{1}+\cdots+c_{a}=l+a-s, c_{1}, \ldots, c_{a} \geq 1} \frac{B_{c_{1}} \cdots B_{c_{a}}}{c_{1} \cdots c_{a}} \\
& =\delta_{s, l}+\sum_{1 \leq a \leq s-1}\left(\begin{array}{l}
s \\
a
\end{array}\right)_{c_{1}+c_{2}+\cdots+c_{a}=l+a-s, c_{1}, \ldots, c_{a} \geq 1} \frac{B_{c_{1}} \cdots B_{c_{a}}}{c_{1} \cdots c_{a}}
\end{aligned}
$$

Assume that $\Lambda_{r, m}=0$ for some integers $r, m$ with $m \geq r \geq 1$. Then we have the following. $\sum_{c_{1}+c_{2}+\cdots+c_{r}=m, c_{1}, \ldots, c_{r} \geq 1} \frac{1}{c_{1} \cdots c_{r}} B_{c_{1}}(\langle x\rangle) \cdots B_{c_{r}}(\langle x\rangle)$ has the Fourier series expansion

$$
\begin{gathered}
\sum_{c_{1}+c_{2}+\cdots+c_{r}=m, c_{1}, \ldots, c_{r} \geq 1} \frac{1}{c_{1} \cdots c_{r}} B_{c_{1}}(\langle x\rangle) \cdots B_{c_{r}}(\langle x\rangle) \\
=C_{0}^{(r, m)}+\sum_{n=-\infty, n \neq 0}^{\infty} C_{n}^{(r, m)} e^{2 \pi i n x}
\end{gathered}
$$

where $C_{0}^{(r, m)}=\sum_{j=1}^{r-1}(-1)^{j-1} \frac{(r)_{j-1}}{m^{j}} \Lambda_{r-j+1, m+1}$, with $C_{0}^{(1, m)}=0$, and $C_{n}^{(r, m)}$, for each $n \neq 0$, are determined recursively from

$$
C_{n}^{(r, m)}=\sum_{j=1}^{m-r+1} \frac{r(m-1)_{j-1}}{(2 \pi i n)^{j}} C_{n}^{(r-1, m-j)}-\sum_{j=1}^{m-r+1} \frac{(m-1)_{j-1}}{(2 \pi i n)^{j}} \Lambda_{r, m-j+1},
$$


and

$$
C_{n}^{(1, m)}=-\frac{(m-1) !}{(2 \pi i n)^{m}}
$$

Here the convergence is uniform.

Next, assume that $\Lambda_{r, m} \neq 0$ for some integers $r, m$ with $m \geq r \geq 1$. Then $\gamma_{r, m}(1) \neq \gamma_{r, m}(0)$. Hence $\gamma_{r, m}(\langle x\rangle)$ is piecewise $C^{\infty}$ and discontinuous with jump discontinuities at integers. Then the Fourier series of $\gamma_{r, m}(\langle x\rangle)$ converges pointwise to $\gamma_{r, m}(\langle x\rangle)$ for $x \notin \mathbb{Z}$ and converges to

$$
\begin{aligned}
\frac{1}{2}\left(\gamma_{r, m}(0)+\gamma_{r, m}(1)\right) & =\gamma_{r, m}(0)+\frac{1}{2} \Lambda_{r, m} \\
& =\sum_{c_{1}+c_{2}+\cdots+c_{r}=m, c_{1}, \ldots, c_{r} \geq 1} \frac{1}{c_{1} \cdots c_{r}} B_{c_{1}} \cdots B_{c_{r}}+\frac{1}{2} \Lambda_{r, m}
\end{aligned}
$$

for $x \in \mathbb{Z}$.

Now, we can state our second result.

Theorem 4.2 For all integers $s, l$ with $l \geq s \geq 1$, we let

$$
\begin{aligned}
\Lambda_{s, l} & =\sum_{0 \leq a \leq s-1}\left(\begin{array}{l}
s \\
a
\end{array}\right) \sum_{c_{1}+c_{2}+\cdots+c_{a}=l+a-s, c_{1}, \ldots, c_{a} \geq 1} \frac{B_{c_{1}} \cdots B_{c_{a}}}{c_{1} \cdots c_{a}} \\
& =\delta_{s, l}+\sum_{1 \leq a \leq s-1}\left(\begin{array}{l}
s \\
a
\end{array}\right)_{c_{1}+c_{2}+\cdots+c_{a}=l+a-s, c_{1}, \ldots, c_{a} \geq 1} \frac{B_{c_{1}} \cdots B_{c_{a}}}{c_{1} \cdots c_{a}} .
\end{aligned}
$$

Assume that $\Lambda_{r, m} \neq 0$ for some integers $r, m$ with $m \geq r \geq 1$. Let $C_{0}^{(r, m)}, C_{n}^{(r, m)}(n \neq 0)$ be as in Theorem 4.1. Then we have the following.

$$
\begin{aligned}
& C_{0}^{(r, m)}+\sum_{n=-\infty, n \neq 0}^{\infty} C_{n}^{(r, m)} e^{2 \pi i n x} \\
& \quad= \begin{cases}\sum_{c_{1}+c_{2}+\cdots+c_{r}=m, c_{1}, \ldots, c_{r} \geq 1} \frac{1}{c_{1} \cdots c_{r}} B_{c_{1}}(x) \cdots B_{c_{r}}(x) & \text { for } x \notin \mathbb{Z}, \\
\sum_{c_{1}+c_{2}+\cdots+c_{r}=m, c_{1}, \ldots, c_{r} \geq 1} \frac{1}{c_{1} \cdots c_{r}} B_{c_{1}} \cdots B_{c_{r}}+\frac{1}{2} \Lambda_{r, m} & \text { for } x \in \mathbb{Z} .\end{cases}
\end{aligned}
$$

Acknowledgements

The third author is appointed as a chair professor at Tianjin Polytechnic University by Tianjin City in China from August 2015 to August 2019.

\section{Competing interests}

The authors declare that they have no competing interests.

\section{Authors' contributions}

All authors contributed equally to the manuscript and typed, read, and approved the final manuscript.

\section{Author details}

'Department of Mathematics, Texas A\&M University - Kingsville, Kingsville, TX 78363, USA. ${ }^{2}$ Department of Mathematics, Sogang University, Seoul, 121-742, Republic of Korea. ${ }^{3}$ Department of Mathematics, Kwangwoon University, Seoul, 139-701, Republic of Korea. ${ }^{4}$ Department of Mathematics, College of Science, Tianjin Polytechnic University, Tianjin, 300160, China. ${ }^{5}$ Department of Mathematics Education and RINS, Gyeongsang National University, Jinju,

Gyeongsangnamdo 52828, Republic of Korea. 


\section{Publisher's Note}

Springer Nature remains neutral with regard to jurisdictional claims in published maps and institutional affiliations.

Received: 28 February 2017 Accepted: 29 July 2017 Published online: 15 August 2017

\section{References}

1. Bayad, A, Kim, T: Higher recurrences for Apostol-Bernoulli-Euler numbers. Russ. J. Math. Phys. 16(1), 1-10 (2012)

2. Ding, D, Yang, J: Some identities related to the Apostol-Euler and Apostol-Bernoulli polynomials. Adv. Stud. Contemp. Math. (Kyungshang) 20(1), 7-21 (2010)

3. Kim, DS, Kim, T: A note on higher-order Bernoulli polynomials. J. Inequal. Appl. 2013, 111 (2013)

4. Kim, DS, Kim, T, Kim, YH, Lee, S-H: Some arithmetic properties of Bernoulli and Euler numbers. Adv. Stud. Contemp. Math. (Kyungshang) 22(4), 467-480 (2012)

5. Kim, T: Some identities for the Bernoulli, the Euler and Genocchi numbers and polynomials. Adv. Stud. Contemp. Math. 20(1), 23-28 (2015)

6. Kim, T: Some identities for the Bernoulli, the Euler and the Genocchi numbers and polynomials. Adv. Stud. Contemp. Math. (Kyungshang) 20(1), 23-28 (2010)

7. Liu, H, Wang, W: Some identities on the the Bernoulli, Euler and Genocchi polynomials via power sums and alternate power sums. Discrete Math. 309, 3346-3363 (2009)

8. Miki, H: A relation between Bernoulli numbers. J. Number Theory 10(3), 297-302 (1978)

9. Srivastava, HM: Some generalizations and basic extensions of the Bernoulli, Euler and Genocchi polynomials. Appl. Math. Inf. Sci. 5(3), 390-414 (2011)

10. Washington, LC: Introduction to Cyclotomic Fields, 2nd edn. Graduate Texts in Mathematics, vol. 83. Springer, New York (1997)

11. Kim, T, Kim, DS, Rim, S-H, Dolgy, D-V: Fourier series of higher-order Bernoulli functions and their applications. J. Inequal. Appl. 2017, 8 (2017)

12. Marsden, JE: Elementary Classical Analysis. Freeman, San Francisco (1974)

13. Zill, DG, Cullen, MR: Advanced Engineering Mathematics. Jones \& Bartlett, Boston (2006)

14. Faber, C, Pandharipande, R: Hodge integrals and Gromov-Witten theory. Invent. Math. 139(1), 173-199 (2000)

15. Kim, DS, Kim, T: Bernoulli basis and the product of several Bernoulli polynomials. Int. J. Math. Math. Sci. 2012, Article ID 463659 (2012)

16. Kim, DS, Kim, T: Some identities of higher order Euler polynomials arising from Euler basis. Integral Transforms Spec. Funct. 24(9), 734-738 (2013)

17. Dunne, GV, Schubert, C: Bernoulli number identities from quantum field theory and topological string theory Commun. Number Theory Phys. 7(2), 225-249 (2013)

18. Gessel, IM: On Miki's identities for Bernoulli numbers. J. Number Theory 110(1), 75-82 (2005)

19. Shiratani, K, Yokoyama, S: An application of p-adic convolutions. Mem. Fac. Sci., Kyushu Univ., Ser. A, Math. 36(1), 73-83 (1982)

\section{Submit your manuscript to a SpringerOpen ${ }^{\circ}$ journal and benefit from:}

- Convenient online submission

- Rigorous peer review

- Open access: articles freely available online

- High visibility within the field

- Retaining the copyright to your article

Submit your next manuscript at $\boldsymbol{~ s p r i n g e r o p e n . c o m ~}$ 